\title{
DIAGNÓSTICO ECOGRÁFICO DE LA CORIONICIDAD Y AMNIONICIDAD EN EL EMBARAZO MÚLTIPLE
}

\author{
Enrique Damián Gil Guevara'; Enrique Gastón Gil Mejía²
}

\begin{abstract}
RESUMEN
La determinación ecográfica de la corionicidad y de la amnionicidad constituye la piedra angular en el manejo obstétrico del embarazo múltiple. Ésta debe hacerse en el primer trimestre del embarazo no solamente por la mayor facilidad diagnóstica sino sobretodo porque constituye el inicio de la ruta a seguir en el control de este tipo de gestaciones. Por lo mencionado anteriormente, el profesional que lidie con esta clase de ecografías debe disponer de los conocimientos necesarios para llegar a un diagnóstico certero de la corionicidad y amnionicidad por ultrasonido.
\end{abstract}

Palabras claves: Corionicidad; Amnionicidad; Ultrasonido; Primer trimestre (Fuente: DeCS BIREME).

\section{DIAGNOSIS BY ULTRASOUND OF THE CHORIONICITY AND AMNIONICITY IN THE MULTIPLE PREGNANCY}

\begin{abstract}
Determination of the chorionicity and amnionicity by ultrasound is the most important step in the management of the multiple pregnancies. This diagnosis should be done in the first trimestre of the pregnancy, not only because of a less difficult process but also because it constitutes the beginning of several steps to follow in the control of these types of pregnancies. All this makes highly necessary that professionals dealing with this kind of ultrasounds have the right knowledge to assess correctly and promptly the chorionicity and amnionicity in multiple pregnancies.
\end{abstract}

Keywords: Chorionicity, amnionicity, ultrasound, first trimester (Source: MeSH NLM).

\section{INTRODUCCIÓN}

El diagnóstico temprano de la corionicidad y amnionicidad forma la base del abordaje moderno del embarazo múltiple. Sin saber estos parámetros básicos, es virtualmente imposible manejar adecuadamente una gestación múltiple.

Por lo tanto, debe quedar claro que tanto la determinación de la corionicidad y de la amnionicidad así como la edad gestacional, no sólo es más exacta, sino también más fácil en el primer trimestre. Asimismo, el diagnóstico temprano del embarazo múltiple permite al gineco-obstetra hacer una explicación oportuna de los riesgos tanto maternos como fetales.

\section{DETERMINACIÓN DE LA CORIONICIDAD Y AMNIONICIDAD EN EL PRIMER TRIMESTRE DEL EMBARAZO}

El embarazo múltiple en el primer trimestre es mucho mejor evaluado en función de la edad gestacional. Por ende, debe basarse en el número de sacos coriónicos, contando embriones o fetos, y el número de corazones que laten, así como finalmente evaluando la naturaleza de los sacos amnióticos y coriónicos ${ }^{1}$.

\section{DETERMINACIÓN DE LA CORIONICIDAD}

Los sacos coriónicos se implantan y son detectados por ultrasonido en uno de los lados de la cavidad central dentro de una decidua apropiadamente gruesa. Esto se logra por el levantamiento de una de las capas endometriales ya sea de las pared anterior o posterior del útero.

Los sacos aparecen como estructuras sonolucentes, redondeadas, flanqueadas por un doble anillo ecogénico que viene a ser el corion².

Estos sacos coriónicos pueden ser detectados desde una edad gestacional tan temprana como las 4 a 5 semanas y simplemente contándolos podemos determinar si la gestación es dicoriónica, tricoriónica o multicoriónica ${ }^{3}$.

Cabe aclarar que, con respecto al término "saco", saco coriónico es el término correcto basado en una nomenclatura embriológica, ya que saco gestacional es un término ultrasonográfico que puede referirse tanto a saco coriónico como amniótico ${ }^{4}$.

Médico Gineco-Obstetra Subespecialista en Medicina Materno-Fetal, King's College Hospital, Londres, Reino Unido.

Médico Gineco-Obstetra Subespecialista en Medicina Materno-Fetal, Universidad Nacional Mayor de San Marcos, Lima, Perú

Recibido: 15-06-15 Aprobado: 12-08-15 


\section{DETERMINACIÓN DEL NUMERO DE EMBRIONES}

A las 6 semanas, por ecografía transvaginal, se puede visualizar tanto los sacos vitelinos como los embriones. Sin embargo, usar solamente el número de sacos coriónicos y el número de sacos vitelinos para determinar el número de embriones puede ser incorrecto. Por lo tanto, es aconsejable esperar a que los latidos cardíacos sean visibles a las 6 semanas de gestación (35 a 42 días postmenstruación) ${ }^{5}$. Cabe aclarar que a esta edad gestacional, el saco vitelino está localizado en el espacio extraembrionario.

El número de sacos vitelinos es usado para determinar amnionicidad en gemelos monocoriónicos en las primeras semanas de gestación aunque esto no constituye una regla general ${ }^{6,7}$.

Finalmente, en caso de gestaciones de mayor orden como cuádruples o quíntuples, el uso de la ecografía 3D ha demostrado una ventaja superior al $2 \mathrm{D}$ disminuyendo notablemente la variabilidad interobservador (en modo 3D surface-rendering).

\section{LAS MEMBRANAS COMO MARCADORES DE CORIONICIDAD Y AMNIONICIDAD}

En una gestación dicoriónica diamniótica, los corion adyacentes crean una estructura fácilmente reconocible referida en la literatura por más de 20 años como el signo "lambda" o "delta" (ver Figura 1) $8,9,10$.

A pesar del número de corions en una gestación múltiple dada, este signo lambda siempre será visto fácilmente entre dos sacos coriónicos adyacentes si el examen ecográfico no es pospuesto más allá de las 16 semanas $^{10}$ La situación es completamente diferente para la gestación monocoriónica diamniótica. En este caso, los dos sacos amnióticos adyacentes obliteran el espacio extraembrionario de tal forma que los amnios se tocan entre ellos y forman una membrana intergemelar relativamente delgada (ver Figura 1) dando como resultado la típica imagen en " $T$ " invertida ${ }^{10}$.

En el caso raro de gestaciones monocoriónicas monoamnióticas, desde las 8 semanas de gestación ya se puede determinar la ausencia de la membrana intergemelar y la presencia de un solo saco vitelino.

En estos casos, es importante descartar la posibilidad de siameses especialmente cuando los embriones o fetos asumen una posición paralela en la misma dirección rostrocaudal. En estas circunstancias, tenemos que determinar si los embriones o fetos se mueven alejándose el uno del otro para excluir que se trate de gemelos siameses ${ }^{11,12}$.

Asimismo, numerosos estudios resaltan la eficacia de la evaluación temprana y simple de la corionicidad y amnionicidad en las primeras 14 semanas.

Por ejemplo, Monteagudo et al. estudiaron 212 gestaciones

(64 gemelos, 87 triples, 41 cuádruples, 18 quíntuples, una gestación de séxtuples y una de séptuples) ${ }^{13}$.

En todas las gestaciones monocoriónicas, el número de sacos vitelinos se correspondía con el número de fetos. Adicionalmente, no se encontró ninguna gestación monocoriónica monoamniótica.

Por otro lado, Bromley y Benacerraf concluyeron que el número de sacos vitelinos visualizados se correspondía con el número de amnios y que dicho número podía ser identificado al menos 2 semanas antes que el número de amnios.

Para concluir con todo lo mencionado anteriormente, podemos decir que:

- La corionicidad puede ser evaluada desde las cinco semanas de gestación.

- El número de embriones con latido cardíaco puede ser determinado a las seis semanas de gestación.

- La amnionicidad puede ser evaluada correctamente a partir de la octava semana de gestación.

- Si cada saco coriónico contiene un solo saco vitelino y un solo embrión con actividad cardiaca, la amnionicidad equivale a la corionicidad (dicoriónico diamniótico, tricoriónico triamniótico, etc.)

- Si cada saco coriónico contiene dos sacos vitelinos y dos embriones con actividad cardiaca, tenemos dos posibilidades, o bien el número de amnios es mayor al numero de corions (monocoriónico diamniótico), o el número de amnios y corions es el mismo (monocoriónico monoamniótico) lo cual es raro pero en este caso tendríamos que esperar al menos hasta la octava semana cuando los amnios son claramente visibles.

- Si un saco coriónico contiene un solo saco vitelino y dos fetos con actividad cardiaca, luego la amnionicidad y la corionicidad son iguales (monocoriónicos monoamnióticos).

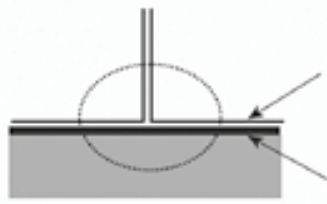

Signo de la "T" invertida

Resumiendo:

$\begin{array}{ll} & \text { Gestación Monocorial } \\ <11 \text { sem } & 2 \text { sacos amnióticos } \\ & 2 \text { sacos vitelinos } \\ & 1 \text { espacio extracelómico } \\ \text { 11-16 sem } & \text { Signo T invertida (Figura 2b) } \\ >16 \text { sem } & \end{array}$

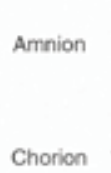

Chorion

$$
\text { (n) }
$$

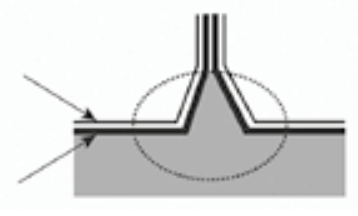

signo "Lambda
Figura 1. Diagnóstico ecográfico de corionicidad
Gestación Bicorial

2 sacos amnióticos

2 sacos vitelinos

2 corions envolvente Signo lambda o delta (Figura 2a)

Sexo fetal discordante 


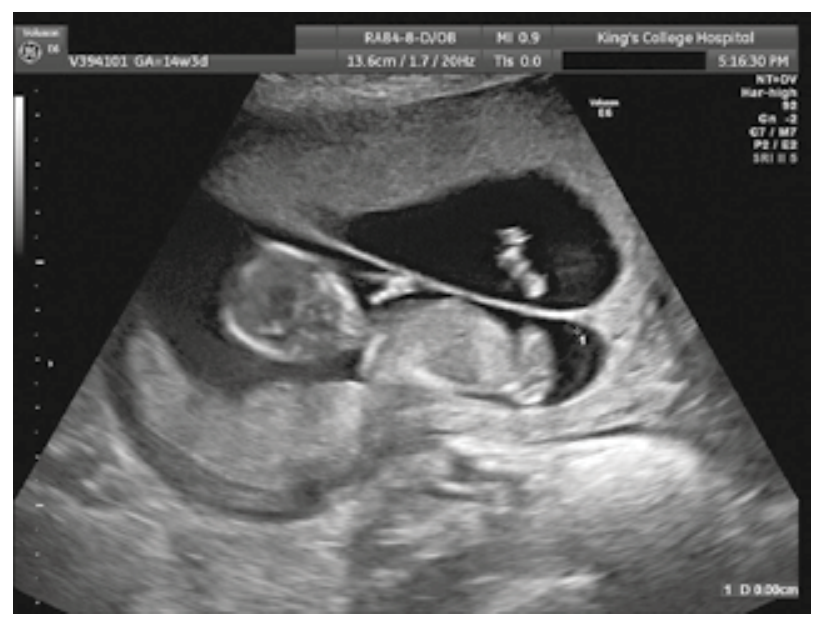

Figura 2a. Diagnóstico ecográfico de corionicidad

Figura 2a). Gestación bicorial de 14 semanas: signo "delta". La presencia de corion entre las dos membranas amnióticas resulta en la gestación bicorial en un signo característico en forma de "V"invertida, o delta griega.

\section{SEGUNDO Y TERCER TRIMESTRES}

Una evaluación adecuada en el primer trimestre permite que los controles del segundo y tercer trimestres sean más relevantes así como también más simples y cortos.

De tal forma que evaluar la amnionicidad y la corionicidad durante el segundo y tercer trimestres, cuando no se hizo en el primer trimestre, puede presentar serios desafíos incluso para manos expertas en ultrasonido.

Los tres determinantes básicos de corionicidad y amnionicidad en el segundo y tercer trimestre son el sexo fetal, la ubicación placentaria y las membranas interfetales.

En circunstancias comunes, los gemelos de sexos diferentes son siempre dicigóticos y por lo tanto siempre dicoriónicos ${ }^{14}$. Por lo tanto, el primer paso en la determinación de la corionicidad y amnionicidad en el segundo y tercer trimestres consiste en evaluar el sexo de cada feto ${ }^{15}$.

De ser el mismo para ambos, el siguiente paso es evaluar las placentas (aspecto y localización) ${ }^{16,17}$. Si se observa dos placentas localizadas en distintas paredes del útero, cada una con su respectiva inserción de cordón umbilical, la gestación es dicoriónica ${ }^{18,19}$, lo que suele ocurrir en un tercio de las gestaciones dobles.

De ver una sola masa placentaria, podría tratarse de una sola placenta o de dos placentas distintas fusionadas, por lo que el siguiente paso constituye evaluar las membranas interfetales.

La determinación de la presencia de la membrana intergemelar es sumamente importante ya que su

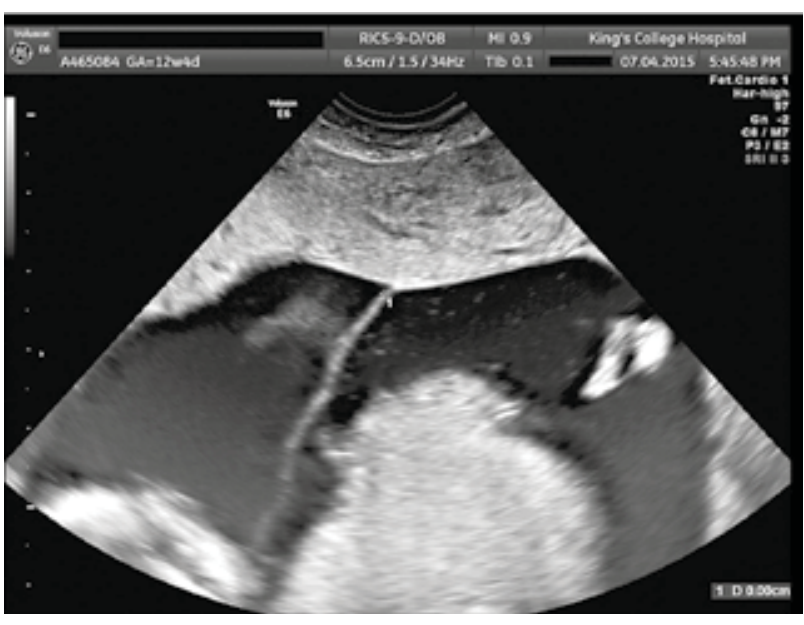

Figura 2b. Diagnóstico ecográfico de corionicidad

Figura 2b). Gestación monocorial de 12 semanas: signo de la " $T$ ". La ausencia de corion entre las dos membranas amnióticas configura una forma de " $T$ ".

ausencia puede cambiar el manejo clínico de una gestación gemelar.

Si no se logra identificar la membrana intergemelar, debemos sospechar o bien que se trata de una gestación monocoriónica monoamniótica o bien de una gestación monocoriónica diamniótica complicada con un síndrome de transfusión feto fetal severo ${ }^{20}$.

Podemos concluir que si se logra identificar dos placentas de localización distinta, ya no es necesario examinar en detalle las membranas interfetales. Sin embargo, en casos en que un solo lecho placentario es identificado, lo siguiente es importantísimo en la evaluación de la corionicidad y amnionicidad ${ }^{17}$ :

- El origen de la membrana intergemelar.

- El grosor de la membrana intergemelar.

- El número de capas presentes en la membrana intergemelar.

\section{Identificar el origen de la membrana intergemelar}

Como se discutió anteriormente, el signo "lambda" es un indicador confiable de dicorionicidad, pero su ausencia no la descarta. Por otro lado, en la gestación monocoriónica, el signo de la "T"' invertida es el signo más confiable de monocorionicidad $^{20}$.

\section{Determinar el grosor de la membrana intergemelar}

Determinar el grosor de la membrana y el número de capas presentes por medio de ultrasonido depende de la frecuencia del transductor, la orientación de la membrana con respecto al transductor y la experiencia del ecografista ${ }^{20}$. 
A mayor frecuencia del transductor, mejor resolución de imagen. Asimismo, en gestaciones avanzadas el usar frecuencias bajas para mejorar la penetración de las ondas de ultrasonido pone en riesgo la correcta identificación de las capas de la membrana intergemelar.

Adicionalmente, la orientación de lamembranaintergemelar en relación al transductor es clave. La membrana debe estar orientada siempre perpendicularmente a las ondas de ultrasonido y así tomar ventaja de la resolución axial del ecógrafo.

En gemelos dicoriónicos, las membranas opuestas son siempre gruesas ya que la membrana intergemelar está compuesta de 4 capas, formada por la combinación del corion y amnios de cada gemelo. Por lo tanto, membranas intergemelares gruesas midiendo $2 \mathrm{~mm}$ o más tienen un valor predictivo positivo entre 89 y $95 \%$ para dicorionicidad $^{18}$ (ver Figura 3).

Por otro lado, una membrana delgada midiendo menos de $2 \mathrm{~mm}$ tiene un VPP de hasta $82 \%$ para monocorionicidad. Sin embargo, esta membrana puede ser difícil de medir ya que suele ser del grosor de un cabello.

Finalmente, cabe aclarar que todo lo anterior se dificulta como es previsto conforme avanza la gestación ${ }^{20}$.

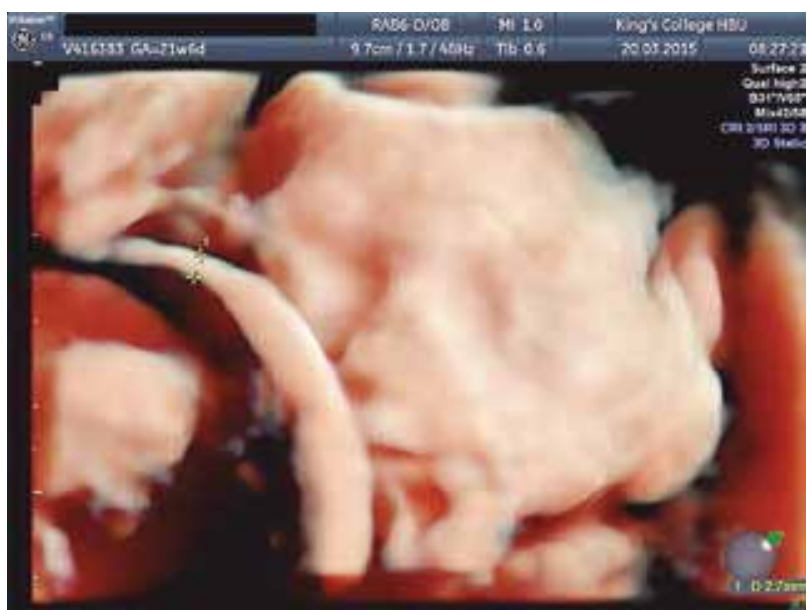

Figura 3. Medición del grosor de la membrana intergemelar. Gestación bicorial de 22 semanas donde se puede apreciar que la medición del grosor de la membrana intergemelar es mayor a $2 \mathrm{~mm}$ en la parte más angosta que a su vez es la parte que se muestra perpendicular a los haces de ultrasonido (orientación correcta).

\section{Contar las capas de la membrana intergemelar}

Las membranas en una gestación dicoriónica diamniótica tienen 4 capas: amnio y corion del primer gemelo, corion y amnio del segundo gemelo; mientras que las de una gestación monocoriónica diamniótica tienen 2 capas: el amnio de cada gemelo.

Por ende, todo lo anterior puede ser visualizado con transductores de alta frecuencia y con una orientación perpendicular de la membrana intergemelar con respecto al transductor como ya se explicó anteriormente (ver figura 3).

\section{REFERENCIAS BIBLIOGRÁFICAS}

1. Kurtz A, Wapner R, Mata J, Johnson A, Morgan P. Twin pregnancies: accuracy of first-trimester abdominal US in predicting chorionicity and amnionicity. Radiology 1992; 185 : 759-62. DOI: 10.1148/radiology.185.3.1438758

2. Goldstein SR. Early pregnancy scanning with endovaginal probe. Contemp Obstet Gynecol 1988; 27: 54-64.

3. Goldstein SR, Snyder J, Watson C, Danon M. Very early pregnancy detection with endovaginal ultrasound. Obstet Gynecol 1988; 72: 200-4. PMID: 3292977

4. Warren W, Timor-Tritsch IE, Peisner D, Raju S, Rosen M. Dating the pregnancy by sequential appearance of embryonic structures. Am J Obstet Gynecol 1989; 161: 747-53. DOI: 10.1016/0002-9378(89)90394-3

5. Monteagudo A, Timor-Tritsch IE, Sharma S. Early and simple determination of chorionic and amniotic type in multifetal gestations in the first fourteen weeks by high frequency transvaginal ultrasonography. Am J Obstet Gynecol 1994; 170: 824-9. DOI: 10.1016/S0002-9378(94)70291-8

6. Doubilet PM, Benson CB. Appearing twin: undercounting of multiple gestations on early first trimester sonograms. J Ultrasound Med 1998; 17: 199-203.

7. PMID: 9544601

8. Bromley B, Benacerraf B. Using the number of yolk sacs to determine amnionicity in early first trimester monochorionic twins. J Ultrasound Med 1995; 14: 415-19. PMID: 7658507

9. Hill LM, Chenevey P, Hecker J, Martin JG. Sonographic determination of first trimester twin chorionicity and amnionicity. J Clin Ultrasound 1996; 24: 305-8. DOI: $10.1002 /(\mathrm{SICl}) 1097-0096(199607 / 08) 24: 6<305:: A I D-$ JCU4>3.0.CO;2-M

10. Bessis R, Papiernik E. Echographic imagery of amniotic membranes in twin pregnancies. En: Gedda K, Parisi P, eds. Twin Research Vol 3: Twin Biology and Multiple Pregnancy. 1981; 11: 571-7.

11. Finberg $\mathrm{H}$. The twin peak sign: reliable evidence of dichorionic twinning. J Ultrasound Med 1992; 11: 571-7. PMID: 1433464

12. Overton TG, Denbow ML, Duncan KR, Fisk NM. First trimester cord entanglement in monoamniotic twins. Ultrasound Obstet Gynecol 1999; 13: 140-2. DOI: 10.1046/j.14690705.1999.13020140.x

13. Copperman AB, Kattenbacher L, Walker B et al. Early firsttrimester ultrasound provides a window through which the chorionicity of twins can be diagnosed in an in vitro fertilization (IVF) population. J Assist Reprod Genet 1995; 12: 693-7.

14. DOI: $10.1007 / \mathrm{BF} 02212895$

15. Monteagudo A, Timor Tritsch 1E, eds. Ultrasound and Multifetal Pregnancy. Carnforth, UK: Parthenon Publishing, 1988.

16. Bronshtein M, Rottem S, Yoffe N, Blumenfeld Z, Brandes JM. Early determination of fetal sex using transvaginal sonography: technique and pitfalls. J Clin Ultrasound 1990; 18: 302-6. DOI: 10.1002/jcu.1870180414

17. Shalev E, Weiner E, Zuckerman H. Ultrasound determination of fetal sex. Am J Obstet Gynecol 1981; 141: 582-3. PMID: 7294083

18. Scardo JA, Ellings JM, Newman RB. Prospective determination of chorionicity, amnionicity and zygosity in twin 
gestations. Am J Obstet Gynecol 1995; 173: 1376-80. DOI: 10.1016/0002-9378(95)90619-3

19. Monteagudo A, Timor-Tritsch IE. Second and third trimester ultrasound evaluation of chorionicity and amnionicity in twin pregnancy. A simple algorithm. J Reprod Med 2000; 45: 47680. PMID: 10900581

20. Mahoney BS, Filly RA, Callen PW. Amnionicity and chorionicity in twin pregnancies: prediction using ultrasound. Radiology 1985; 155: 205-9.

21. DOI: http://10.1148/radiology.155.1.3883418

22. Westover T, Perry R, Dunh T, Fischer R. Prospective assessment of placental cord insertion in twin gestation. Am J Obstet Gynecol 1997; 176: S74.
23. DOI: $10.1016 / \mathrm{S} 0002-9378(97) 80299-2$

24. Eixarch E, Goncé A, Gratacós E. Gestación Gemelar. En: Figueras F, Gratacós E, Puerto B. Actualización en Medicina MaternoFetal. $1^{\text {ra }}$ ed. Barcelona: Ergon; 2015. Loc. 78058353.

Correspondencia: Dr. Enrique Gil Guevara Harris Birthright Research Centre, King's College Hospital SE5 9RS, Londres, Reino Unido +447787210184

enrique.gil@doctors.org.uk 